\title{
A GENERAL FORM OF 2D FOURIER TRANSFORM EIGENFUNCTIONS
}

\author{
Soo-Chang Pei ${ }^{1}$ and Chun-Lin Liu ${ }^{2}$ \\ Department of Electrical Engineering ${ }^{1}$ \\ Graduate Institute of Communication Engineering ${ }^{1,2}$ \\ National Taiwan University, Taipei, Taiwan, 10617 \\ Email: pei@cc.ee.ntu.edu.tw ${ }^{1}$,r99942052@ntu.edu.tw ${ }^{2}$
}

\begin{abstract}
In this paper, the general form of the two-dimensional Fourier transform (2D FT) eigenfunctions is discussed. It is obtained from the linear combination of the 2D separable Hermite Gaussian functions (SHGFs). For example, the rotated Hermite Gaussian functions (RHGFs) for the rotated coordinate and the Laguerre Gaussian functions (LGFs) for the polar coordinate are two special cases of the general form. With the aid of the general form, we can achieve these 2D functions with perfect orthogonality. Finding the combination coefficients is equivalent to the multinomial expansion problem. Therefore, we can apply the fast Fourier transform and some recurrence relations to the coefficients. The computation cost is much less than the close-form coefficients, which is associated with the Jacobi polynomials.
\end{abstract}

Index Terms - Two-Dimensional Discrete Fourier Transforms, Eigenfunctions, Orthogonality, Fast Fourier Transforms.

\section{INTRODUCTION}

For a 1D signal $f(x) \in L^{2}(\mathbb{R})$, the 1D FT is a transformation from $f(x)$ to $F(u)$ and defined by

$$
F(u)=\mathcal{F}\{f(x)\}=\frac{1}{\sqrt{2 \pi}} \int_{-\infty}^{\infty} f(x) e^{-j u x} d x .
$$

The eigenfunctions of (1) are the Hermite Gaussian functions (HGFs),

$$
h_{n}(x)=\left(2^{n} n ! \sqrt{\pi}\right)^{-1 / 2} H_{n}(x) e^{-x^{2} / 2},
$$

where $H_{n}(x)=(-1)^{n} e^{x^{2}}(d / d x)^{n} e^{-x^{2}}$ is the Hermite polynomial. Substituting (2) into (1) yields

$$
\mathcal{F}\left\{h_{n}(x)\right\}=(-j)^{n} h_{n}(u) .
$$

$\left\{h_{n}(x)\right\}$ forms a complete orthonormal basis set of $L^{2}(\mathbb{R})$. The eigenfunctions and the eigenvalues are important to define the fractional Fourier transform [1].
The 2D FT, similar to the 1D FT, is defined by

$$
\begin{aligned}
F(u, v) & =\mathcal{F}\{f(x, y)\} \\
& =\frac{1}{2 \pi} \int_{-\infty}^{\infty} \int_{-\infty}^{\infty} f(x, y) e^{-j(u x+v y)} d x d y .
\end{aligned}
$$

The eigenfunctions of (4) are not as simple as (2). There are mainly three complete orthonormal basis sets: separable Hermite Gaussian functions (SHGFs), rotated Hermite Gaussian functions (RHGFs) and Laguerre Gaussian functions (LGFs).

\subsection{Separable Hermite Gaussian functions}

The SHGFs are obtained by multiplying the HGFs in different dimensions,

$$
h_{m, n}(x, y)=h_{m}(x) h_{n}(y) .
$$

Substituting (5) into (4) gives us

$$
\mathcal{F}\left\{h_{m, n}(x, y)\right\}=(-j)^{m+n} h_{m, n}(u, v) .
$$

Because of the complete and orthonormal property of the HGFs, $\left\{h_{m, n}(x, y)\right\}$ is also a complete orthonormal basis set of $L^{2}\left(\mathbb{R}^{2}\right)$.

\subsection{Rotated Hermite Gaussian functions}

The RHGFs are also the eigenfunctions of the 2D FT because of the rotation theorem [2],

$$
\begin{aligned}
& \mathcal{F}\{f(x \cos \alpha+y \sin \alpha,-x \sin \alpha+y \cos \alpha)\} \\
= & F(u \cos \alpha+v \sin \alpha,-u \sin \alpha+v \cos \alpha),
\end{aligned}
$$

where $\alpha$ is the rotation angle in the counterclockwise direction. If we combine (6) and (7), we obtain

$$
\mathcal{F}\left\{h_{m, n}(\alpha ; x, y)\right\}=(-j)^{m+n} h_{m, n}(\alpha ; u, v),
$$

where

$h_{m, n}(\alpha ; x, y)=h_{m, n}(x \cos \alpha+y \sin \alpha,-x \sin \alpha+y \cos \alpha)$

are the RHGFs. Note that the eigenvalues of the RHGFs are the same as those of the SHGFs. 


\subsection{Laguerre Gaussian functions}

For the circular symmetric signals, the LGFs $l_{m, n}(r, \theta)$ are the eigenfunctions of the 2D FT in the polar coordinate $(r, \theta)$, which is known as the Hankel transform (HT). In [3], the eigenfunctions of the HT are

$$
l_{m, n}(r, \theta)=N_{p, l} r^{l} L_{p}^{l}\left(r^{2}\right) e^{-r^{2} / 2} e^{-j l \theta},
$$

where $p=\min \{m, n\}, l=|m-n|, N_{p, l}$ is the normalization factor, and $L_{n}^{m}(\cdot)$ is the associated Laguerre polynomial. Substituting (10) into (4) gives us the eigenvalues, $(-j)^{m+n}$.

There are other eigenfunctions instead of the above three families. However, these functions are rare and they usually do not form an orthonormal basis. Besides, the above three sets of eigenfunctions form a complete and orthonormal basis, which means that any eigenfunction can be uniquely determined from the basis. Hence, we focus ourselves on constructing the basis rather than the special cases.

\section{THE GENERAL FORM OF THE EIGENFUNCTIONS}

The three types of functions form a complete and orthonormal basis set relatively. Therefore, we can use all of the SHGFs as basis and proper combination coefficients to synthesis the RHGFs or the LGFs. It is observed that all solutions contain two parts: the 2D Gaussian function and the particular polynomial. As a result, we assume that the eigenfunctions are in the form of

$$
\psi^{D}(x, y)=P^{D}(x, y) e^{-\left(x^{2}+y^{2}\right) / 2},
$$

where $P^{D}(x, y)$ is a two variable polynomial with degree $D$. From the degree property of the Hermite polynomials, i.e. $\operatorname{deg}\left(H_{n}(x)\right)=n, P^{D}(x, y)$ can be expressed as

$$
P^{D}(x, y)=\sum_{p+q \leq D} \frac{c_{p, q}^{D}}{\sqrt{2^{p+q} p ! q ! \pi}} H_{p}(x) H_{q}(y) .
$$

The coefficients $c_{p, q}^{D}$ are unique once $P^{D}(x, y)$ is fixed. Then

$$
\psi^{D}(x, y)=\sum_{p+q \leq D} c_{p, q}^{D} h_{p, q}(x, y) .
$$

Taking the 2D FT on both sides of (13) yields

$$
\mathcal{F}\left\{\psi^{D}(x, y)\right\}=\sum_{p+q \leq D}(-j)^{p+q} c_{p, q}^{D} h_{p, q}(u, v) .
$$

Compare (13) with (14) and we obtain that the $p$ and $q$ must satisfy

$$
p+q=D, D-4, D-8, \ldots
$$

and $p, q \geq 0$. The above result is explained in an intuitive way as follows. The eigenfunctions satisfying (15) correspond to the same eigenvalue. The linear combination of these bases is still the eigenfunctions of the 2D FT.

Although the coefficients $c_{p, q}^{D}$ can be arbitrarily chosen, we also want the obtained eigenfunctions to be complete and orthonormal. Assume that the two eigenfunctions are $\psi_{1}^{D}(x, y)$ and $\psi_{2}^{D^{\prime}}(x, y)$, which are specified by the coefficients $c_{p, q}^{D}$ and $d_{p, q}^{D^{\prime}}$, as in (13), respectively. The inner product of the two eigenfunctions becomes

$$
\left\langle\psi_{1}^{D}(x, y), \psi_{2}^{D^{\prime}}(x, y)\right\rangle=\sum_{p, q} c_{p, q}^{D}\left(d_{p, q}^{D^{\prime}}\right)^{*},
$$

where $(\cdot)^{*}$ denotes complex conjugation. To simplify (16), we restrict $p+q=D$ in $\psi_{1}^{D}(x, y)$ and $p+q=D^{\prime}$ in $\psi_{2}^{D^{\prime}}(x, y)$ so that (13) can be revised as

$$
\psi^{D}(x, y)=\sum_{p=0}^{D} c_{p, D-p}^{D} h_{p, D-p}(x, y)
$$

and (16) becomes 0 if $D \neq D^{\prime}$. For $D=D^{\prime}$, there are $D+1$ terms in (16), which can be viewed as the inner product of two $(D+1)$-dim vectors. (17) is considered to be the general form of the 2D FT eigenfunctions.

It is possible to choose appropriate $c_{p, q}^{D}$ and $d_{p, q}^{D}$ to make the coefficients orthonormal. One trivial solution is that

$$
c_{p, q}^{D}=\delta[p, q-D], \quad d_{p, q}^{D}=\delta[p-1, q-D+1] .
$$

The obtained solutions are two of the SHGFs. We further conclude that Any $(D+1)$-dim orthonormal vector set is the possible solution of the coefficients $c_{p, q}^{D}$ and $d_{p, q}^{D}$. The RHGFs and LGFs are in the form of (13) with proper coefficients. To have $h_{m, n}(\alpha ; x, y)$, the coefficients are [4]

$$
\begin{aligned}
c_{p, q}^{m, n}(\mathrm{RHGF})= & \sqrt{\frac{p ! q !}{m ! n !}}(\sin \alpha)^{m-p}(\cos \alpha)^{n-p} \\
& P_{p}^{(m-p, n-p)}(\cos 2 \alpha)
\end{aligned}
$$

for $p+q=m+n=D . P_{n}^{(\alpha, \beta)}(x)$ are the Jacobi polynomials. In [5], the close-form coefficients of the LGFs are similar to those of the RHGFs as long as the rotation matrix $\mathbf{U}$ is modified into UZ. Another more elegant form comes from the Laguerre Gaussian modes of the paraxial wave equation $[4,6]$. The $\pi / 4$ RHGFs and LGFs are connected with a complex factor $j^{k}$ in the coefficients.

The major advantage of the general form is the orthogonality of the eigenfunctions. The above derivation is under the assumption that the HGFs are continuous. If the discrete HGFs, as the eigenvectors of the DFT, with perfect orthogonality are used, the obtained discrete 2D signals are the eigenfunctions of the 2D DFT and they are orthonormal, using the same coefficients $c_{p, q}^{m, n}$.

The general form (17) combines $h_{p, D-p}(x, y)$. Note that the sum of the subscripts is a constant. Following the same step, we also find that this property is correct for higher dimensions. For instance, the 3D FT eigenfunctions are the linear combination of $h_{p, q, r}(x, y, z)$ with $N=p+q+r$. 


\section{FFT ALGORITHM FOR THE COEFFICIENTS}

The close-form combination coefficients, in (19), are not suitable for computation. To obtain a single $c_{p, q}^{m, n}$, there are at least $p$ multiplications because the degree of the Jacobi polynomial is $p$. For all $c_{p, q}^{m, n}, p=0,1, \ldots, m+n$, the number of multiplications required is $O\left(D^{2}\right)$, which can be reduced.

In [5], the combination coefficients $c_{p, q}^{m, n}$ are derived from the definition of the Hermite 2D polynomials by

$$
\begin{aligned}
H_{m, n}(U ; x, y) & =2^{m+n} e^{-\frac{1}{4}\left(\frac{\partial^{2}}{\partial x^{2}}+\frac{\partial^{2}}{\partial y^{2}}\right)} \\
& \left(U_{x x} x+U_{x y} y\right)^{m}\left(U_{y x} x+U_{y y} y\right)^{n},
\end{aligned}
$$

which is (3.4) in [5]. If we set $\left\{U_{x x}, U_{x y}, U_{y x}, U_{y y}\right\}=$ $\{\cot \alpha, \sin \alpha,-\sin \alpha, \cos \alpha\}$ and multiply the Gaussian function, (20) becomes $h_{m, n}(\alpha ; x, y)$. By expanding the multinomial and some derivation, we have the coefficients associated with the Jacobi polynomials, as shown in (19). From the point of computing, expanding the multinomial (20) is faster than evaluating (19) directly because the multinomial expansion problem is equivalent to the discrete convolution. Assume that the result of expansion is

$$
\left(U_{x x} x+U_{x y} y\right)^{m}\left(U_{y x} x+U_{y y} y\right)^{n}=\sum_{p, q} a_{p, q}^{m, n} x^{p} y^{q} .
$$

Our goal is to get $a_{p, q}^{m, n}$ from the left-hand side. if we set $x=e^{-j 2 \pi /(D+1)}$ and $y=1$, the right-hand side is exactly the DFT while the left-hand side involves multiplication in the DFT domain. Taking the inverse DFT yields

$$
\left[U_{x y}, U_{x x}\right]^{* m} *\left[U_{y y}, U_{y x}\right]^{* n}=\left[a_{0, D}^{m, n}, \ldots a_{D, 0}^{m, n}\right],
$$

where $*$ denotes the convolution and $(\cdot)^{* n}=(\cdot) * \ldots *(\cdot)$ for $n$ times. We can use FFT algorithms to compute the left-hand side of (22) and the results are the coefficients $a_{p, q}^{m, n}$. The final coefficients $c_{p, q}^{m, n}$ is related to $a_{p, q}^{m, n}$ by a normalization factor $\sqrt{p ! q ! /(m ! n !)}$. Therefore, $c_{p, q}^{m, n}$ can be obtained faster with the aid of the FFT algorithms.

The complexity of this fast algorithm is approximately $O\left((D+1) \log _{2}(D+1)\right)$. First, computing the DFT of $\left[U_{x y}, U_{x x}\right]$ and $\left[U_{y y}, U_{y x}\right]$ takes about $(D+1) \log _{2}(D+1)$ complex multiplications if $(D+1)$-point Cooley-Tukey FFT algorithm is applied. Second, the power of $m$ and $n$ in the frequency domain requires approximately $(D+1) \log _{2}(m n)$ complex multiplications and the inverse DFT takes another $\frac{1}{2}(D+1) \log _{2}(D+1)$ complex multiplications. Therefore, the overall system complexity is $O\left((D+1) \log _{2}(D+1)\right)$, which outperforms the method for computing the Jacobi polynomials in (19) directly.

This algorithm can be applied to the combination coefficients of the LGFs. In [5], the LGFs are closely related to the RHGFs by introducing a complex matrix $\mathbf{Z}$ and modifying the values $U_{x x}, U_{x y}, U_{y x}, U_{y y}$ into complex values. The other process remains.
Another advantage of this algorithm is that if we want to obtain the coefficients of $c_{p, q}^{m, n}$ for all $m, n, p, q$, the results can be obtained recursively. From (22), we have

$$
\begin{aligned}
& {\left[a_{0, D}^{m, n}, \ldots, a_{D, 0}^{m, n}\right] *\left[U_{x y}, U_{x x}\right]=\left[a_{0, D+1}^{m+1, n}, \ldots, a_{D+1,0}^{m+1, n}\right],} \\
& {\left[a_{0, D}^{m, n}, \ldots, a_{D, 0}^{m, n}\right] *\left[U_{y y}, U_{y z}\right]=\left[a_{0, D+1}^{m, n+1}, \ldots, a_{D+1,0}^{m, n+1}\right] .}
\end{aligned}
$$

The above recursive relation is useful when we want to implement the whole set of the orthonormal functions.

\section{EXPERIMENTAL RESULTS}

In this section, some properties of the eigenfunctions are discussed and compared with the continuous-sampled functions. The discrete 1D HGFs are computed with perfect orthogonality [7]. We select the number of discrete sample in one dimension to be $N=101$ and the sampling interval $\Delta_{x}=$ $\Delta_{y}=\sqrt{2 \pi / N}$ for simplicity.

In Table 1 , the RHGF $h_{8,3}(\pi / 3 ; x, y)$ and the LGF $l_{8,3}(x, y)$ are shown in two methods. The continuous-sampled functions are taken from (9) and (10) while the discrete functions are obtained by our method. The latter really approximates the continuous function we want, in terms of the magnitude and the phase. With the aid of the general form, we do not have to re-sample and the sample points are fixed.

In Table 2, three of the 2D DFT eigenfunctions are listed in our method. The eigenfunctions $\psi(x, y)$, with magnitude and phase, are shown in the first row while in the second row, their Fourier transforms $\mathcal{F} \psi$ are given. Due to the eigenvalues, $(-j)^{m+n}$, the magnitudes are invariant but there are constant phase differences between $\psi(x, y)$ and $\mathcal{F} \psi$. Some mismatches in the phase information near the boundaries are due to small magnitudes (below the order of $10^{-16}$ ), resulting in inaccurate phase. The first one is $h_{4,10}(3 \pi / 4 ; x, y)$, whose the phase difference is $\pi$, consisting with our simulation. For $l_{7,6}(x, y)$, the phase difference is $-\pi / 2$ theoretically. In our simulation, the phase pattern rotates $\pi / 2$ clockwise. The third function is generated from the random complex coefficients $c_{p, q}^{D}$ with $D=12$. The phase difference between $\psi(x, y)$ and $\mathcal{F} \psi$ should be perfectly zero, which is verified by our simulation. The function seems very complicated, but it is still the 2D DFT eigenfunction.

\section{CONCLUSION AND FUTURE WORK}

In this paper, the general form of the eigenfunctions of the 2D DFT was discussed. The three common 2D FT eigenfunctions, SHGFs, RHGFs and LGFs, are all special cases of the general form in (17), which can also be extended to higher dimensions. A fast algorithm utilizing the FFT algorithm, with complexity $O\left((D+1) \log _{2}(D+1)\right)$, was proposed. Based on the discrete 1D HGFs and the coefficients, the 2D eigenfunctions are obtained and they are indeed the eigenfunctions of the 2D DFT. 


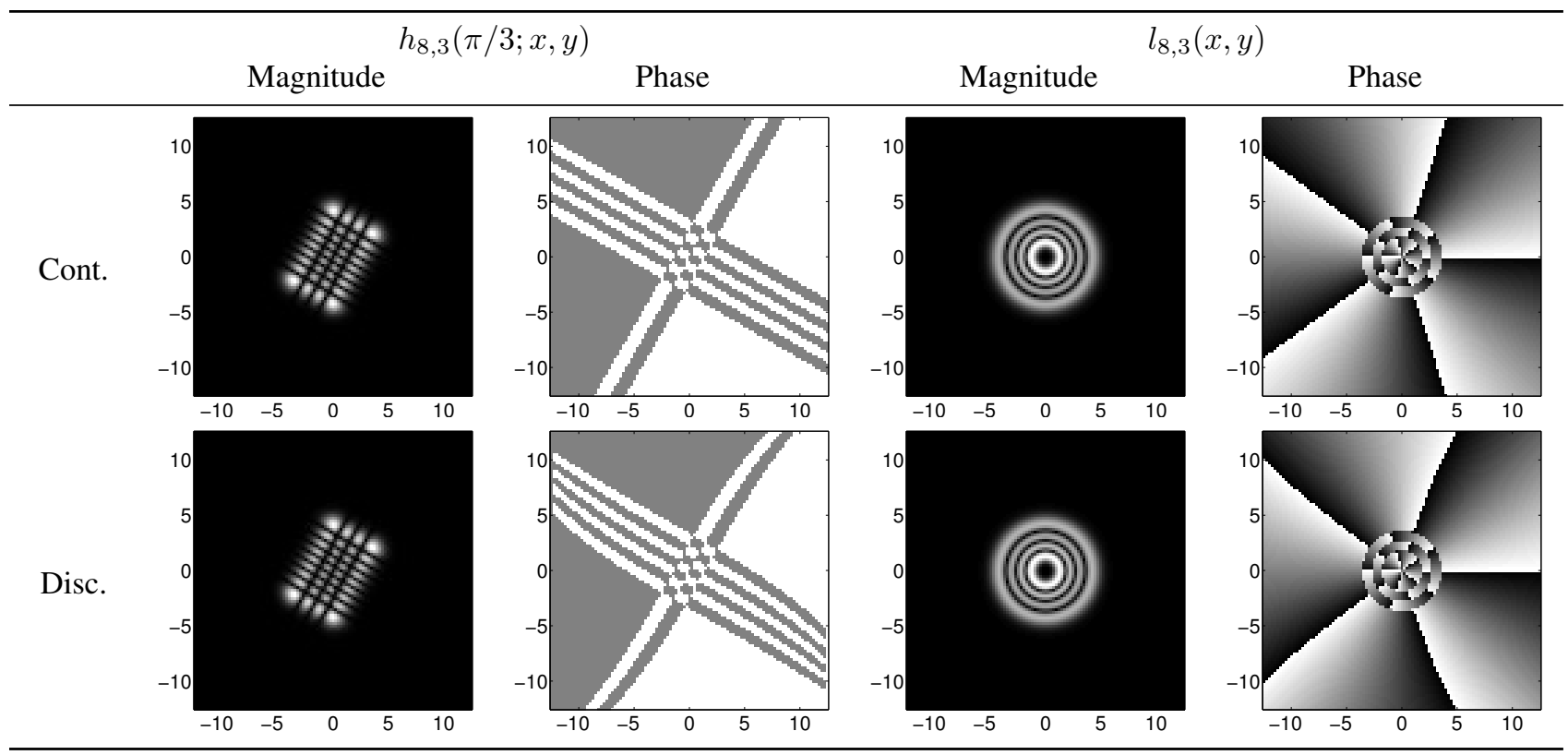

Table 1. The comparison between the continuous-sampled functions/discrete functions of $h_{8,3}(\pi / 3 ; x, y)$ and $l_{8,3}(x, y)$.

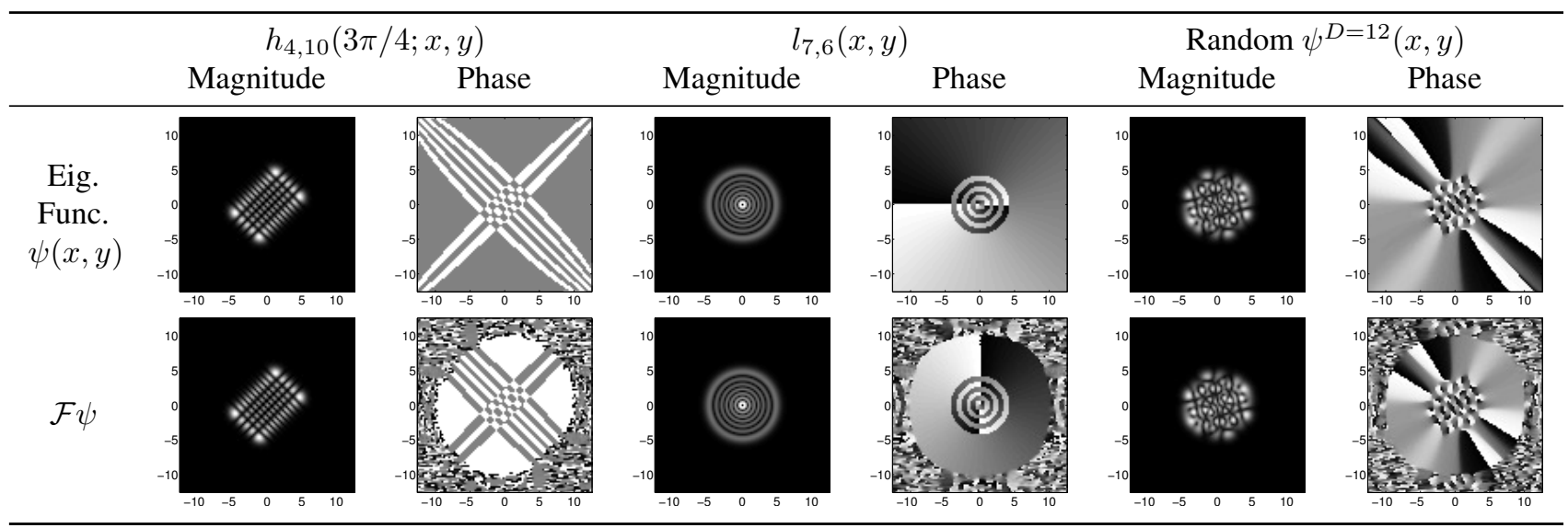

Table 2. The comparison between the eigenfunctions and their Fourier transforms.

These eigenfunctions can be used to define the corresponding transforms to analyze rotated or circular symmetric objects. Besides, our method is free from interpolation and ensures orthogonality. It is more suitable for discrete signals.

\section{REFERENCES}

[1] V. Namias, "The fractional order Fourier transform and its application to quantum mechanics," J. Inst. Maths Applics, vol. 25, pp. 241-265, 1980.

[2] R. N. Bracewell, The Fourier Transform and Its Applications, McGrawHill, 3 edition, 2002.

[3] V. Namias, "Fractionalization of Hankel transforms," $J$. Inst. Maths Applics, vol. 26, pp. 187-197, 1980.
[4] E. G. Abramochkin and V. G. Volostnikov, "Generalized Hermite-Laguerre-Gauss beams," Physics of Wave Phenomena, vol. 18, pp. 14-22, 2010.

[5] A Wünsche, "General Hermite and Laguerre twodimensional polynomials," J. Phys. A: Math. Gen., vol. 33, pp. 1603-1629, 2000.

[6] M. W. Beijersbergen, L. Allen, H.E.L.O. van der Veen, and J. P. Woerdman, "Astigmatic laser mode converters and transform of orbital angular momentum," Opt. Comm., vol. 96, pp. 123-132, 1993.

[7] F. A. Grünbaum, "The eigenvectors of the discrete Fourier transform: A version of the Hermite functions," J. Math. Anal. Appl., vol. 88, pp. 355-363, 1982. 